\title{
In-vivo imaging of nanoshell extravasation from solid tumor vasculature by photoacoustic microscopy
}

Meng-Lin Li, Jon A. Schwartz, James Wang, George Stoica, Lihong V. Wang

Meng-Lin Li, Jon A. Schwartz, James Wang, George Stoica, Lihong V. Wang, "In-vivo imaging of nanoshell extravasation from solid tumor vasculature by photoacoustic microscopy," Proc. SPIE 6437, Photons Plus Ultrasound: Imaging and Sensing 2007: The Eighth Conference on Biomedical Thermoacoustics, Optoacoustics, and Acousto-optics, 64370B (28 February 2007); doi: 10.1117/12.697722

SPIE. Event: SPIE BiOS, 2007, San Jose, California, United States 


\title{
In vivo imaging of nanoshell extravasation from solid tumor vasculature by photoacoustic microscopy
}

\author{
Meng-Lin Li ${ }^{\mathrm{a}}$, Jon A. Schwartz ${ }^{\mathrm{b}}$, James Wang ${ }^{\mathrm{b}}$, George Stoica ${ }^{\mathrm{c}}$, and Lihong V. Wang ${ }^{\mathrm{a}}$ \\ aptical Imaging Laboratory, Department of Biomedical Engineering, Texas A\&M University, \\ College Statoin, Texas, USA 77843-3120 \\ ${ }^{b}$ Nanospectra Biosciences, Inc., Houston, Texas, USA 77054 \\ ${ }^{c}$ Department of Veterinary Pathobiology, Texas A\&M University, College Station, Texas, USA \\ $77843-5547$
}

Email: $\underline{\text { mlli@ee.nthu.edu.tw, } 1 \text { hwang@,biomed.wustl.edu }}{ }^{\dagger}$

\begin{abstract}
In this study, high resolution reflection-mode (backward-mode) photoacoustic microscopy (PAM) is used to noninvasively image progressive extravasation and accumulation of nanoshells within a solid tumor in vivo. This study takes advantage of the strong near-infrared absorption of nanoshells, a novel type of optically tunable gold nanoparticles that tend to extravasate from leaky tumor vasculatures (i.e., passive targeting) via the "enhanced permeability and retention" effect due to their nanoscale size. Tumors were grown in immunocompetent BALB/c mice by subcutaneous inoculation of CT26.wt murine colon carcinoma cells. PEGylated nanoshells with a peak optical absorption at $\sim 800 \mathrm{~nm}$ were intravenously administered. Pre-scans prior to nanoshell injection were taken using a 584-nm laser source to highlight blood content and an 800-nm laser source to mark the background limit for nanoshell accumulation. After injection, the three-dimensional nanoshell distribution inside the tumor was monitored by PAM for 7 hours. Experimental results show that nanoshell accumulation is heterogeneous in tumors: more concentrated within the tumor cortex and largely absent from the tumor core. This correlates with others' observation that drug delivery within tumor cores is ineffective because of both high interstitial pressure and tendency to necrosis of tumor cores. Since nanoshells have been recently applied to thermal therapy for subcutaneous tumors, we anticipate that PAM will be important to this therapeutic technique.
\end{abstract}

Keywords: photoacoustic microscopy, nanoshell extravsation, enhanced permeability and retention effect

\section{INTRODUCTION}

Contrast-agent-aided optical imaging techniques have high sensitivity and specificity. Pure optical imaging, however, faces a major challenge-the overwhelming optical scattering in biological tissue; as a result, its spatial resolution significantly decreases with increasing imaging depth. Photoacoustic imaging, a novel hybrid imaging modality, which combines the merits of both light and ultrasound, has been applied to measuring the optical properties of biological tissues noninvasively ${ }^{1}$. It has been shown a promising tool for visualizing tissue structures and functions with high optical contrast, good spatial resolution, and satisfactory imaging depth ${ }^{2}$.

Recently, an in vivo backward-mode confocal photoacoustic microscope (PAM) with dark-field illumination was invented by Maslov et al. to image blood vessels in the skin ${ }^{3}$. This system offers high lateral resolution (45 $\mu \mathrm{m}$ at the focal point) and high axial resolution $(\sim 15 \mu \mathrm{m})$, and it is capable of imaging optical absorption contrast as deeply as 3 $\mathrm{mm}$ in biological tissue. This PAM system shows potential for applications in dermatology and related cancer research.

\footnotetext{
${ }^{\dagger}$ Lihong V. Wang and Meng-Lin Li are currently with Optical Imaging Laboratory, Department of Biomedical Engineering, Washington University in St. Louis, and Department of Electrical Engineering, National Tsing Hua University, Taiwan, respectively.
} 
Our research goal for this study is to develop a nanoparticle-based technique which integrates photoacoustic imaging and photothermal therapy for seamless detection and treatment of solid tumors. The nanoparticle we used here is gold nanoshell, which trade name is AuroShell ${ }^{\mathrm{TM}}$, provided by Nanospectra Biosciences, Inc. A gold nanoshell is a new kind of optically tunable nanoparticle, consisting a silica core surrounded by a thin gold layer. By adjusting the size of the nanoparticle core relative to the thickness of the gold shell, gold nanoshells can absorb various wavelengths of light ${ }^{4}$. Recently, gold nanoshells have been applied to photothermal therapy for subcutaneous murine tumors ${ }^{5}$, and also have been used as a contrast agent for in vivo brain images using photoacoustic tomography, in which gold nanoshells enhance the image contrast of the cortex vessels in a rat brain ${ }^{6}$.

In this study, we use gold nanoshells as contrast agents of tumor imaging. The PAM is used to noninvasively image progressive extravasation of gold nanoshells from solid tumor vasculature in vivo without any invasive biological modification such as a dorsal skin-fold window chamber ${ }^{7}$. Nanoshells tend to preferentially extravasate and accumulate in tumors - a preferential, size dependent accumulation - via the "enhanced permeability and retention" effect due to their nanoscale size (i.e., passive targeting) ${ }^{5,8}$. We anticipate that the integration of PAM into gold-nanoshell based photothermal therapy to characterize and monitor the accumulation of gold nanoshells in solid tumors would be an invaluable aid.

\subsection{Gold nanoshell}

\section{METHODS}

Gold nanoshells are a new type of optically tunable particle composed of a dielectric core (silica, $\sim 125 \mathrm{~nm}$ in diameter in our case) coated with an ultra-thin gold shell (10-12 nm in our case). The optical properties of nanoshells depend dramatically on the relative sizes of the core and the thickness of the gold shell. By varying the relative core size and shell thicknesses, the optical properties of nanoshells can be varied across a broad range of the electromagnetic spectrum that spans the visible and the infrared regions. Nanoshells can be made either to preferentially absorb or scatter light by varying the absolute size of the particle relative to the wavelength of the light at their optical resonance. In our cancer applications, gold nanoshells designed to absorb near-infrared wavelengths are used. The peak optical absorption of nanoshells is at $\sim 800 \mathrm{~nm}$ (see figure $1^{6}$ ), a wavelength that exhibits optimal penetration into scattering biological tissues and is the isosbestic point of molar extinction spectra of oxy- and deoxy-hemoglobin, thus reducing the effect of blood oxygenation changes on photoacoustic signals. In addition, the gold nanoshells we used here is PEGylated nanoshells. PEGylation prolongs the circulation time. The PEGylated nanoshells were measured to have a half-life of $\sim 3.7 \mathrm{~h}$ in the blood of immune-competent BALB/c mice.

\section{$\underline{2.2}$ System setup}

Figure 2 shows the experimental setup of photoacoustic microscopy for in vivo imaging of nanoshell extravasation in solid tumors. A tunable Ti:Sapphire laser (LT-2211A, Lotis) and a tunable dye laser (ND6000, Continuum) were employed to provide pulses with wavelengths of $800 \mathrm{~nm}$ and $584 \mathrm{~nm}$, respectively. Pre-scans prior to nanoshell injection were taken using a 584-nm laser source to highlight blood content and an 800-nm laser source to mark the background limit for nanoshell accumulation and to monitor the nanoshell accumulation. The laser pulse widths for the Ti:Sapphire and the dye laser were $15 \mathrm{~ns}$ and $6.5 \mathrm{~ns}$, respectively, and the pulse repetition rate was $10 \mathrm{~Hz}$ for both lasers. The laser output was delivered to a multimode fiber with a $0.6 \mathrm{~mm}$ diameter. The tip of the fiber was positioned so as to have a diverging annular beam, focused using a conical lens so that the light focus overlapped with the focus of the high-frequency ultrasound transducer (V214-BC-RM, Panametrics). The incident light energy densities of both the NIR and visible light sources on the sample surface were controlled to be less than 3 and $1 \mathrm{~mJ} / \mathrm{cm}^{2}$, respectively, which are well within the safety standards. An ultrasound transducer with a central frequency of $50 \mathrm{MHz}$, a nominal bandwidth of $70 \%$, and a numerical aperture (NA) of 0.44 was used, which proves a lateral resolution of $45 \mu \mathrm{m}$ and an axial resolution of $15 \mu \mathrm{m}$. The photoacoustic signals received by the transducer were amplified and then recorded by a digital oscilloscope (TDS 5034B, Tektronics). The transducer was immersed in water inside a plastic container with an opening at the bottom that was sealed with a thin transparent disposable membrane. The animal was placed outside the container below the membrane, and the ultrasonic coupling was further secured by coupling gel. A mechanical stage provided lateral scanning of the photoacoustic transducer, which allowed a cross-sectional B-scan and a planar C-scan over the sample to be obtained. 


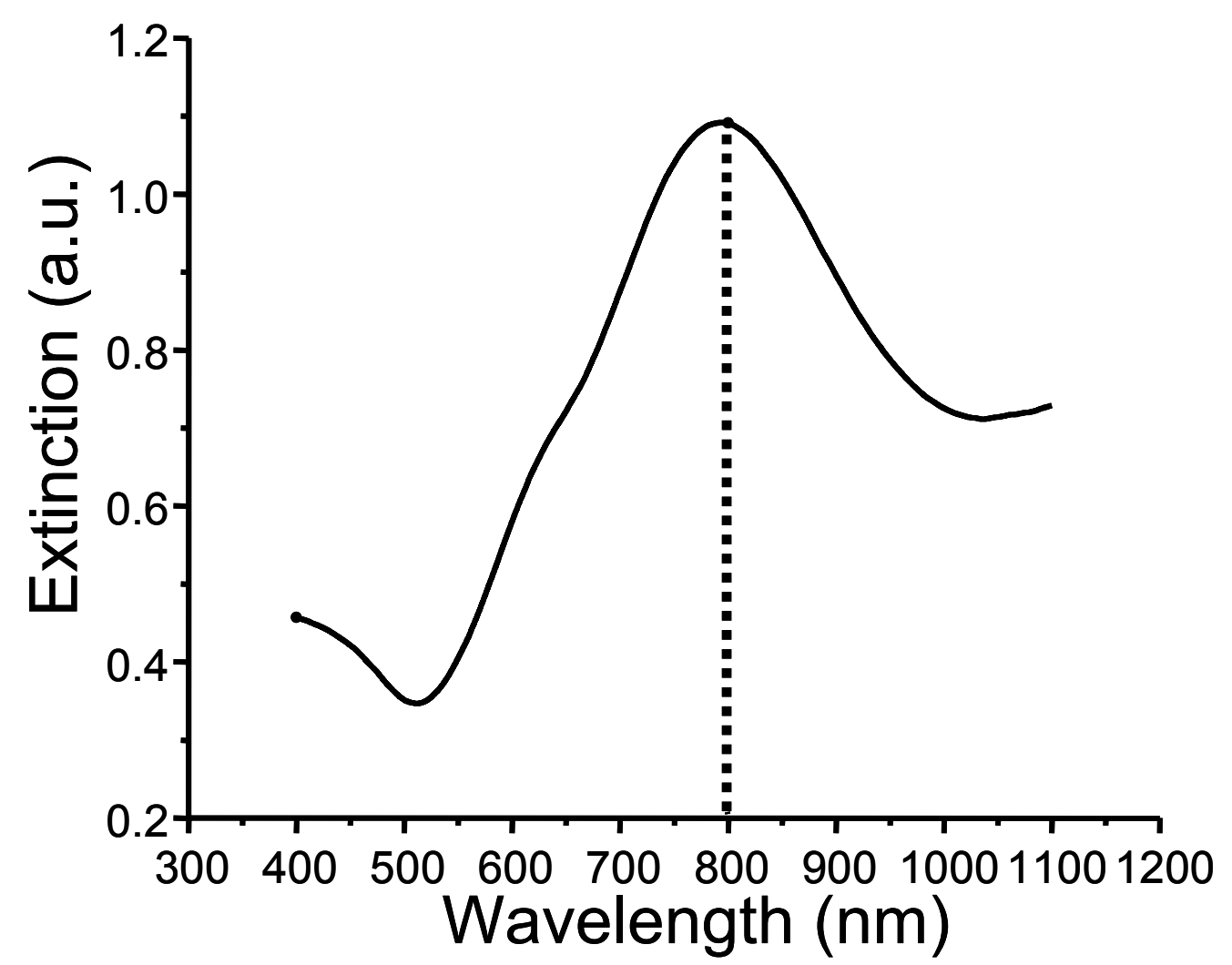

Figure 1. Extinction spectrum of the nanoshell solution, where the absorption peak is about $800 \mathrm{~nm}^{6}$.

\subsection{Animal handling}

Immunocompetent $\mathrm{BALB} / \mathrm{c}$ mice weighing about 20 grams were used for the in vivo animal experiments. The mice were inoculated with CT26.wt murine colon carcinoma cells subcutaneously in the upper lumbar area to the left of the vertebra 7 days before the experiment. Imaging was performed under isoflurane gas anesthesia with a dose of $1 \%$ pure oxygen at a $1 \mathrm{~L} / \mathrm{min}$ flow rate. The body temperature of the animal was maintained by controlling the temperature of the water in the immersion container to $39^{\circ} \mathrm{C}^{7}$. The pulse rate and the global arterial blood oxygenation were monitored during the entire experiment using a pulse oximeter (Model 8600, Nonin Medical Inc.) Pre-scans prior to nanoshell injection were taken using a 584-nm laser source to highlight blood content and an 800-nm laser source to mark the background limit for nanoshell accumulation. PEGylated nanoshells with a peak optical absorption at $\sim 800 \mathrm{~nm}$ were then intravenously administered. The nanoshell dose is about $3 \times 10^{9} \mathrm{NS} / \mathrm{g} \mathrm{BW}\left(\sim 4 \times 10^{10} \mathrm{NS} / \mathrm{mL}\right.$ blood $)$. After injection, the three-dimensional nanoshell distribution inside the tumor was monitored by PAM for 7 hours. After imaging, the mice were sacrificed using pentobarbital $(120 \mathrm{mg} / \mathrm{kg}$, IP). All of the experimental animal procedures were approved by the University Laboratory Animal Care Committee of Texas A\&M University. 


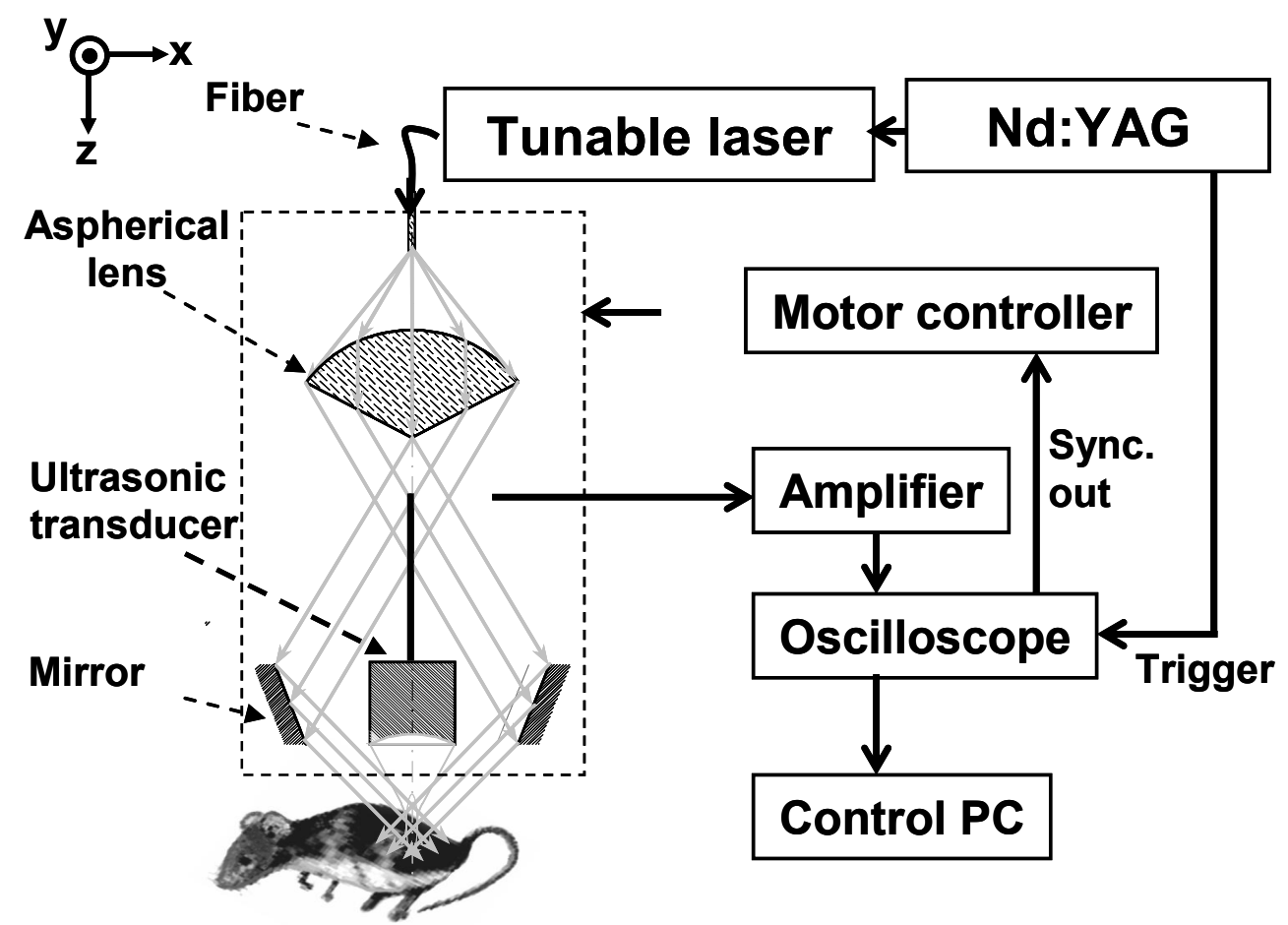

Figure 2. Schematic of the photoacoustic microscope for in vivo imaging of nanoshell extravsation in solid tumors

\section{RESULTS AND DISCUSSION}

PAM measurements illustrate the passive uptake of nanoshells within the cortex of an inoculated CT26.wt tumor. Figures 3 (a) and (b) are, respectively, C-scan images taken prior to nanoshell injection using a $584 \mathrm{~nm}$ laser source to highlight blood content and an $800 \mathrm{~nm}$ laser source to set the background limit for nanoshell accumulation. In $\mathrm{C}$-scan images, the maximum amplitude of each scan line along the depth direction versus the $2 \mathrm{D}$ transducer position is plotted. Figure 3(a) shows the feeding vessels and tumor angiogenesis well. The tumor position is marked by the character "T". In figure 3(b), the bright spots are caused by electronic noise and the interference signals from the scalp surface. Note that without nanoshells, signal amplitudes in the $800 \mathrm{~nm}$ image is about one order smaller than those in the $584 \mathrm{~nm}$ image even though the incident light energy of $800 \mathrm{~nm}$ is about 3 times higher than that of $584 \mathrm{~nm}$. Figures 3(c) and (d) show PAM C-scan images taken using an $800 \mathrm{~nm}$ laser source at 0.5 and 3 hours post nanoshell injection, respectively. Compared with figure 3(b), the nanoshells improve the signal amplitudes by about one order, as indicated by the colorbars in figures 3(b), (c), and (d). In addition, the blood contrast is enhanced. The nanoshell accumulation delineates the tumor contour. Figures 3(c) and (d) illustrate the progressive uptake of nanoshells within the tumor cortex, as shown at the positions indicated by the arrows in figure 3(c). Note that the position of the mouse was slightly shifted toward the right hand side during the administration of nanoshells, which can be observed from figure 3 .

Figures 4(a) shows the B-scan image at $584 \mathrm{~nm}$ before nanoshell injection, of which the scanning position corresponds to the dashed line in figure 3(d). In B-scan images, the vertical axis is the depth and the horizontal axis shows the lateral scanning position. In figure 4(a), the scalp surface is indicated by the dashed line, and the tumor position is marked by the character "T". Figure 4(a) shows the positions of the vessels. Figure 4(b) is the B-scan image taken at $800 \mathrm{~nm}$ prior to nanoshell injection to set the background limit for nanoshell accumulation. Figures $4(\mathrm{c})$ and (d) show PAM B-scan images taken using an $800 \mathrm{~nm}$ laser source at 0.5 and 3 hours post nanoshell injection, respectively. The bright spots, as indicated by the arrow in figures 4 (c) and (d), in these two images are the 
photoacoustic signals from accumulated nanoshells. These two images show the depth information where nanoshells accumulate, which also indicate the nanoshell distributes around the tumor surface, i.e., tumor cortex.

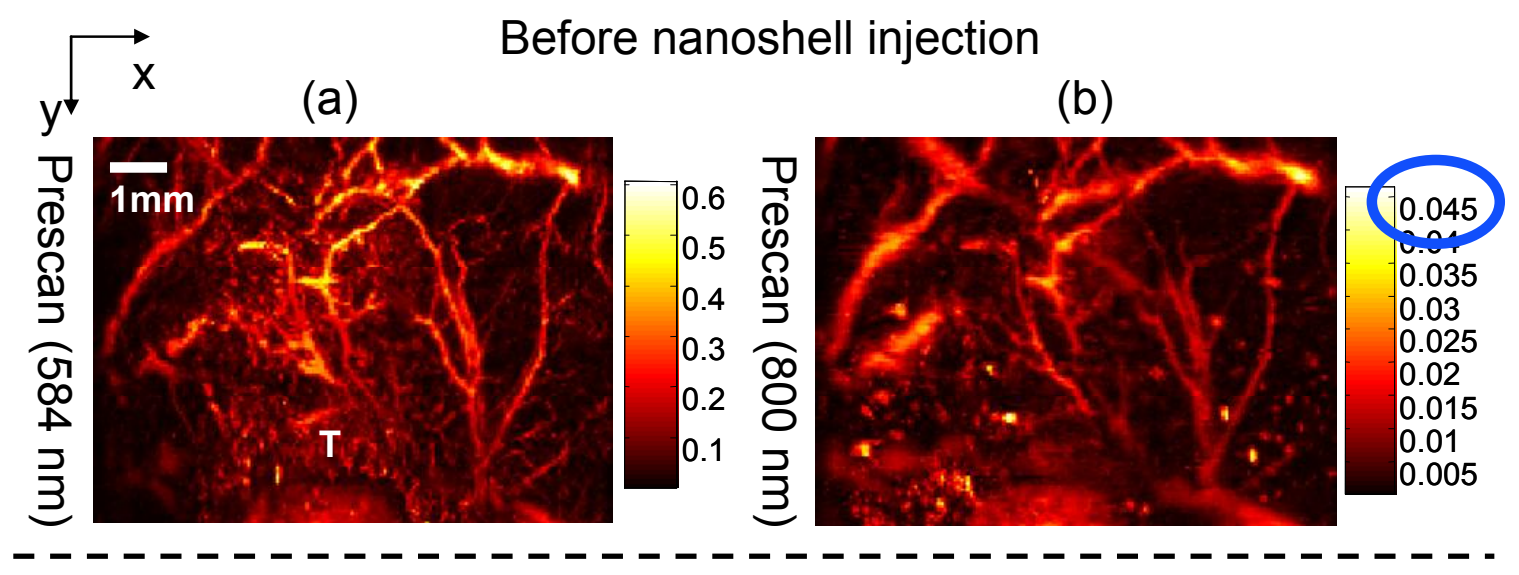

\section{After nanoshell Injection (800 nm)}
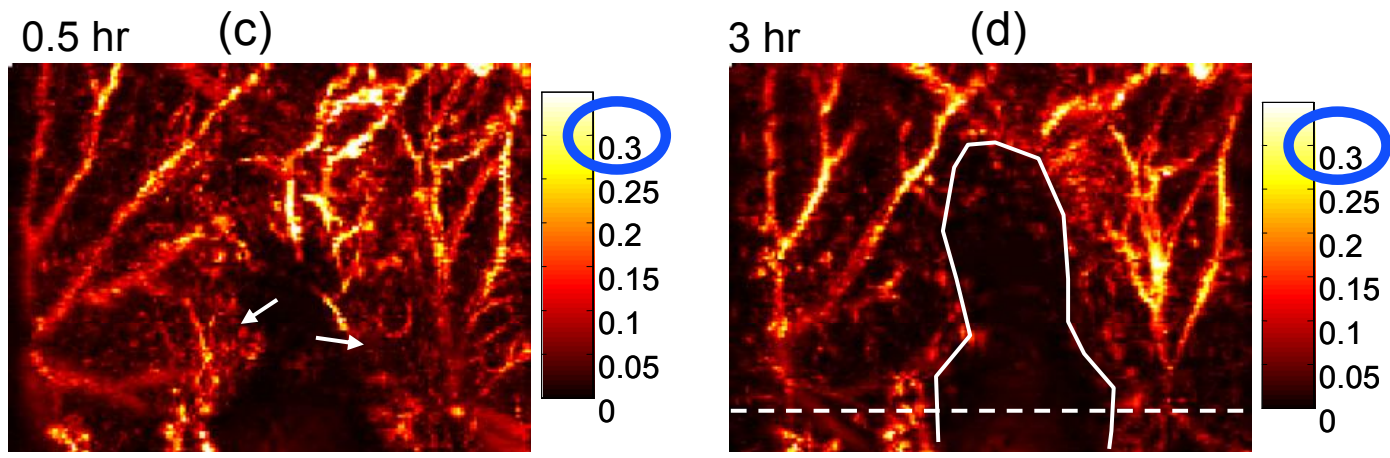

Figure 3. (a) C-scan image at $584 \mathrm{~nm}$ before nanoshell injection to highlight initial blood content; (b) Prescan image (Cscan) at $800 \mathrm{~nm}$ to set pre-nanoshell injection image baseline; (c) and (d) C-scan images of nanoshell accumulation in tumor cortext at 0.5 and 3 hours after nanoshell injection (at $800 \mathrm{~nm}$ ), respectively

An absence of nanoshells within the tumor core is observed in figures 3(c) and (d), and figures 4(c) and (d). It is shown that nanoshell accumulation is heterogeneous in tumors: more concentrated within the tumor cortex and largely absent from the tumor core. These results are further verified by histology. This correlates with others' observation that drug delivery within tumor cores is ineffective because of both high interstitial pressure and tendency to necrosis of tumor cores ${ }^{9,10}$. Moreover, an absence of nanoshells on the top surface of the tumor is also observed. Membrane pressure may preclude accumulation there.

By observing the changes in photoacoustic signals from the vessels and tumor regions, the time-dependent variation of absorption in the vessels and tumor regions attributable to the nanoshells can be studied. We calculated the averaged optical absorption in vessels and tumor regions as a function of time, which represents the clearance profiles of the nanoshells in vessles and tumor regions, respectively. It is shown that nanoshells in vessels are gradually cleared out by the circulatory system of the mouse and nanoshells in tumor regions are progressively uptaken at the tumor site owing to poor lymphatic drainage of the tumor (i.e., the enhanced permeability and retention effect). At $\sim 7$ hours after nanoshell injection, the contrast ratio of the tumor regions to vessels is about 2 to 1. 


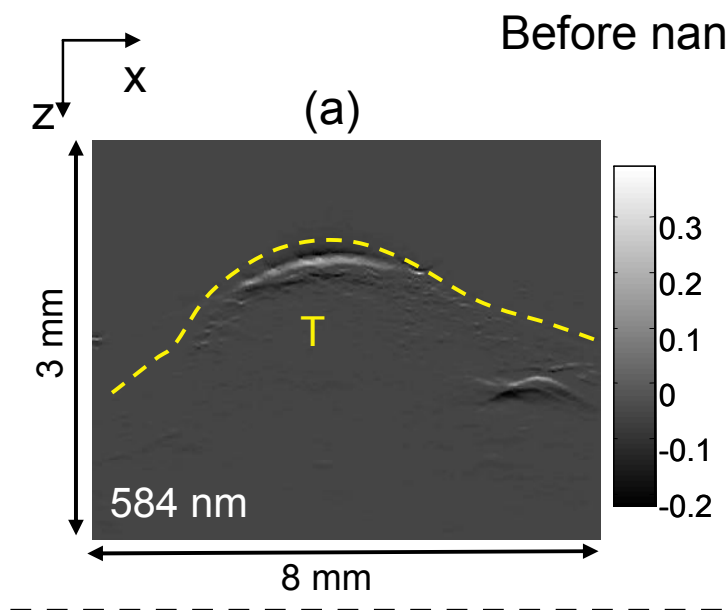

(b)

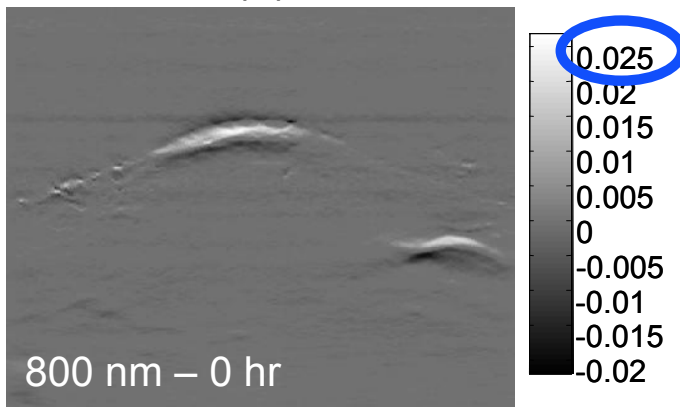

After nanoshell Injection $(800 \mathrm{~nm})$

(c)

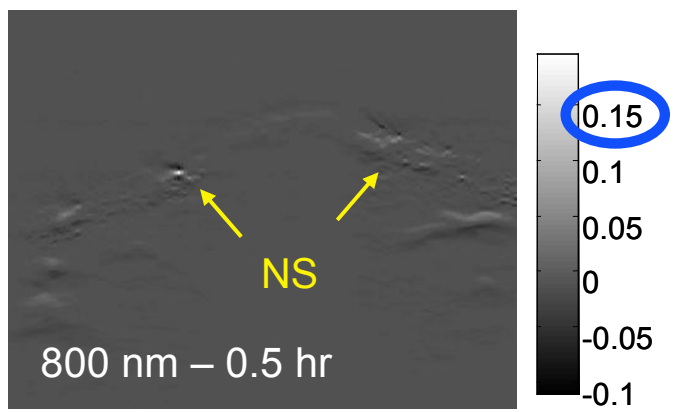

(d)

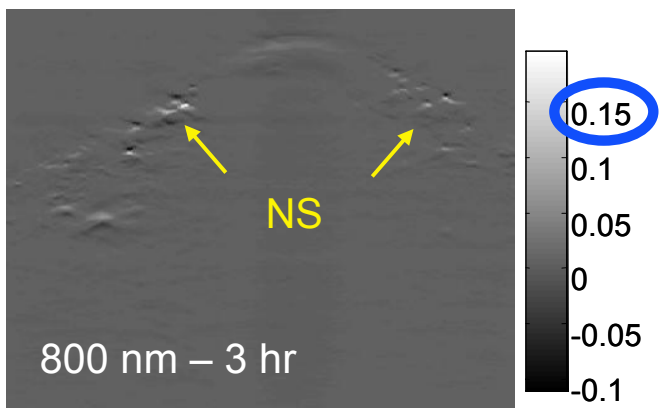

Figure 4. (a) B-scan image at $584 \mathrm{~nm}$ before nanoshell injection to highlight initial blood content; (b) Prescan image (Bscan) at $800 \mathrm{~nm}$ to set pre-nanoshell injection image baseline; (c) and (d) B-scan images of nanoshell accumulation in tumor cortext at 0.5 and 3 hours after nanoshell injection (at $800 \mathrm{~nm}$ ).

\section{CONCLUSIONS}

In summary, by employing the PAM, we successfully imaged extravasation of gold nanoshells, a novel nanoparticle contrast agent, from solid tumor vasculatures in vivo with high spatial resolution and satisfactory sensitivity. It is found that nanoshell accumulation is heterogeneous in tumors: more concentrated within the tumor cortex and largely absent from the tumor core. Using exogenous contrast from nanoshells, image contrast is also significantly enhanced at $800 \mathrm{~nm}$. Since gold nanoshells have been recently applied to photothermal therapy for subcutaneous tumors, we anticipate that PAM will be important to this therapeutic technique to monitor the accumulation of gold nanoshells. In addition, by using nanoshells conjugated to bioactive materials such as proteins, antibodies, and drugs, the technique presented here (PAM plus nanoshells) will potentially enable molecular imaging and therapeutic monitoring ${ }^{11}$.

\section{ACKNOWLEDGEMENTS}

We are grateful to Gina Lungu, Ovidiu Cracium, Kelly L. Gill for assistance with cell culture and veterinary procedure, and to Jung-Taek Oh, Hao F. Zhang, and Konstantin Maslov for technical advice and prior system development. This project is sponsored in part by the National Institutes of Health grants R01 EB000712 and R01 NS46214, and in part by 
the Advanced Technology Program at NIST, cooperative agreement \#70NANB4H3040. Travel support from National Tsing Hua University, Taiwan is also greatly appreciated.

\section{REFERENCES}

1. G. Ku, X. Wang, X. Xie, G. Stoica, and L. V. Wang, "Imaging of tumor angiogenesis in rat rains in vivo by photoacoustic tomography," Appl. Opt. 41, 770-775, 2005.

2. X. Wang, Y. Pang, G. Ku, X. Xie, G. Stoica, and L. V. Wang, "Noninvasive laser-induced photoacoustic tomography for structural and functional in vivo imaging of the brain," Nat. Biotechnol. 21, 803-806, 2003.

3. K. Maslov, G. Stoica, and L. V. Wang, "In vivo dark-field reflection-mode photoacoustic microscopy," Opt. Lett. 30, 625-627, 2005.

4. S. J. Oldenburg, J. B. Jackson, S. L. Westcott, N. J. Halas, "Infrared extinction properties of gold nanoshells," Appl. Phys. Lett., 111, 2897, 1999.

5. D. P. O'Neal, L. R. Hirsch, N. J. Halas, J. D. Payne, and J. L. West, "Photo-thermal tumor ablation in mice using near infrared-absorbing nanoparticles," Cancer Lett., 209, 171-176, 2004.

6 . Y. Wang, X. Xie, and X. Wang et al., "Photoacoustic tomography of a nanoshell contrast agent in the in vivo rat brain," Nano Lett. 4, 1689-1692, 2004.

7. G. Kong, R. D. Braun, and M. W. Dewhirst, "Characterization of the effect of hyperthermia on nanoparticle extravasation from tumor vasculature," Cancer Res. 61, 3027-3032, 2001.

8. X. Gao, Y. Gui, R. M. Levenson, L. W. K. Chung and S. Nie, "In vivo cancer targeting and imaging with semiconductor quantum dots," Nat. Biotechnol. 22, 969-976, 2004.

9. T. Padera, B. Stoll, and J. Tooredman et al., "Cancer cells compress intratumour vessels", Nature 427, 695, 2004.

10. P. Liu, A. Zhang, Y. Xu, and X. Xu, "Study of non-uniform Microparticle liposome extravasation in tumour," Int. J. Hyperthermia, 21, 259-270, 2005.

11. G. Loo, L. Hirsch, and M.-H. Lee et al., "Gold nanoshell bioconjuates for molecular imagingin living cells," Opt. Lett. 30, 1012-1014, 2005 